\title{
Integración de la variación infra-específica de coníferas Mediterráneas en modelos de distribución de especies. Aplicaciones para la evaluación de la vulnerabilidad y la conservación
}

Integrating infra-specific variation of Mediterranean conifers in species distribution models. Applications for vulnerability assessment and conservation

\author{
Serra Varela, M J Jesús ${ }^{1,2,3,{ }^{*}}$
}

${ }^{1}$ Instituto Universitario de Gestión Forestal Sostenible, Universidad de Valladolid (UVa)-INIA, Carretera de la Coruña Km 7.5, 28040, Madrid, España

${ }^{2}$ INIA, Centro de Investigación Forestal, Departamento de Ecología y Genética Forestal Carretera A Coruña km 7.5, 28040 Madrid, España 


\section{Resumen}

La gran amenaza que supone el cambio climático para los sistemas forestales hace necesario desarrollar herramientas que evalúen la vulnerabilidad de las especies que los componen, de cara a guiar la gestión para la conservación y a facilitar la toma de decisiones. Los modelos de distribución de especies son una herramienta útil en este campo a pesar de que tienen ciertas limitaciones derivadas de la falta de incorporación de información genética que impide la consideración de la capacidad adaptativa de las especies, la frecuente no consideración de las interacciones bióticas, que adquiere una gran importancia al poder verse alteradas con los cambios climáticos y la no integración de la incertidumbre ligada a las diferentes predicciones climáticas futuras y sus diferentes escenarios. Por tanto, a lo largo de esta tesis se desarrollan métodos para mejorar la aplicación de los modelos de distribución de especies de cara a la conservación forestal, con el objetivo principal de mantener la capacidad adaptativa de las especies, dada su importancia de cara a lidiar con las alteraciones climáticas.

Palabras clave: Gestión forestal, Mediterráneo, Capacidad adaptativa, Información molecular, Información genética, Interacciones bióticas, Pinus halepensis, Pinus pinaster, Fusarium circinatum.

\section{Summary}

Climate change is threatening forest ecosystems, driving the need to develop tools to assess species' vulnerability, to support conservation management decisions. Species distribution models provide a useful tool to fulfill this challenge although there are some issues that limit their application in forest conservation such as that they rarely incorporate genetic information, of species adaptive capacity, that there are few examples in literature in which SDMs consider biotic interactions although they can be altered by climate change and that most of SDM-based approaches fail in dealing with future uncertainties derived from the wide range of future climate models and scenarios currently available. The main objective of this thesis is to overcome these limitations and thus to improve the applicability of species distribution models for forest conservation considering as a primary goal the maintenance of species adaptive capacity a key element for increasing species' ability to cope with climate change.

Keywords: Forest management, Mediterranean, Adaotive capacity, Molecular information, Genetic information, Pinus halepensis, Pinus pinaster, Fusarium circinatum. 


\section{Introducción}

La gran amenaza que supone el cambio climático para los sistemas forestales hace necesario desarrollar herramientas que evalúen la vulnerabilidad de las especies que los componen, de cara a guiar la gestión para la conservación. Mantener la capacidad adaptativa de las especies (Nicotra et al., 2015) debe ser un objetivo principal para los gestores, dada su importancia para lidiar con las alteraciones climáticas.

En el ámbito de la conservación, los modelos de distribución de especies (MDE; Guisan \& Zimmermann, 2000) son una herramienta útil (Guisan et al., 2013) ya que relacionan la distribución de las especies con variables ambientales pudiendo proyectarse en cualquier marco temporal o geográfico. Sin embargo, hay determinados factores que limitan la aplicabilidad de estos modelos. En primer lugar, hasta ahora, los MDE rara vez incorporan información genética a pesar de la gran cantidad de autores destacando su importancia (Thuiller et al., 2008; Matyas et al., 2009; Thomassen et al., 2010; Schoville et al., 2012), especialmente en el caso de árboles forestales, considerando, a pesar de las evidencias de lo contrario, que todas las poblaciones de una misma especie reaccionan de igual manera ante los cambios climáticos. En segundo lugar, son escasos los ejemplos en los que los MDE consideran factores bióticos (Araújo \& Luoto, 2007) de cara a evaluar la vulnerabilidad de las especies al cambio climático, lo cual es importante ya que los cambios en las condiciones climáticas pueden alterar las interacciones entre especies. Por último, la mayoría de estas aproximaciones no incorporan en sus predicciones la incertidumbre derivada de la gran variedad de modelos y escenarios de clima futuro disponibles (aunque en Fordham et al., 2011 se puede encontrar un ejemplo de lo contrario). Esta tesis, por tanto, pretende integrar estos elementos (información genética, exposición biótica e incertidumbre climática futura) a lo largo de sus distintos capítulos persiguiendo optimizar la aplicación de los MDE para la gestión forestal y para su conservación.

La tesis se estructura en cuatro artículos científicos (ver Tabla 1) en los cuales se lidia con cada uno de los apartados descritos anteriormente. En el primer trabajo, se incorpora a los MDE información molecular y se comprueba que mejora las predicciones. En el segundo, se evalúa la vulnerabilidad al cambio climático de las especies analizadas mediante MDE que ya incorporan información genética para considerar la capacidad adaptativa y evaluando la exposición al cambio climático integrando la incertidumbre derivada de las proyecciones climáticas. La incorporación de la exposición biótica en la evaluación de la vulnerabilidad se analiza en el tercer trabajo. Por último, se aplica lo aprendido para tratar de utilizar los MDE para localizar poblaciones marginales no solo desde el comúnmente evaluado punto de vista geográfico, sino también desde el punto de vista ambiental.

\section{Material y métodos}

Los trabajos se centraron principalmente en el área Mediterránea dada la gran 
biodiversidad característica de sus ecosistemas (Myers et al., 2000), su gran vulnerabilidad y exposición al cambio climático (IPCC, 2007) y su poca representación en otros trabajo de conservación anteriores (Lefèvre et al., 2013). En función de los objetivos de cada trabajo y de los datos disponibles el análisis se realizó para una o varias especies y con una extensión espacial concreta (ver Tabla 1).

Tabla 1. Esquema general de la estructura de la tesis, incluyendo objetico de cada Trabajo, especies modelizadas, marco especial y publicación resultante.

\begin{tabular}{|c|c|c|c|c|}
\hline & Objetivo & $\begin{array}{c}\text { Marco } \\
\text { Espacial }\end{array}$ & $\begin{array}{c}\text { Especie } \\
\text { modelizada }\end{array}$ & Resultados \\
\hline Trabajo 1 & $\begin{array}{l}\text { (i) Testar la influencia de la infor- } \\
\text { mación molecular en los Modelos } \\
\text { de Distribución de Especies. } \\
\text { (ii) Papel del ambiente y la geo- } \\
\text { grafía en la estructura infra-espe- } \\
\text { cífica. }\end{array}$ & $\begin{array}{l}\text { Oeste de la } \\
\text { Cuenca } \\
\text { Mediterránea } \\
\text { Costa Atlántica } \\
\text { Europea }\end{array}$ & Pinus pinaster Ait. & $\begin{array}{l}\text { Serra-Varela } \\
\text { et al., (2015) } \\
\text { Global Ecology } \\
\text { and } \\
\text { Biogrography }\end{array}$ \\
\hline Trabajo 2 & $\begin{array}{l}\text { (i) Evaluación de la vulnerabi- } \\
\text { lidad. } \\
\text { (ii) Selección de las estrategias de } \\
\text { conservación adecuadas basadas } \\
\text { en la exposición al cambio climá- } \\
\text { tico. }\end{array}$ & Europa & $\begin{array}{l}\text { Pinus pinaster Ait.. } \\
\text { Pinus halepensis Mill. }\end{array}$ & $\begin{array}{l}\text { Serra-Varela, } \\
\text { et al., (2017) } \\
\text { Diversity and } \\
\text { Distributions }\end{array}$ \\
\hline Trabajo 3 & $\begin{array}{l}\text { (i) Incorporación de la exposición } \\
\text { biótica en los Modelos de Distri- } \\
\text { bución de Especies. } \\
\text { (ii) Proporcionar una herramienta } \\
\text { útil para la gestión forestal. }\end{array}$ & $\begin{array}{c}\text { España } \\
\text { Peninsular }\end{array}$ & Pinus pinaster Ait. & $\begin{array}{l}\text { Serra-Varela, } \\
\text { et al., (2017) } \\
\text { PlosOne }\end{array}$ \\
\hline Trabajo 4 & $\begin{array}{l}\text { (i) Delimitación de poblaciones } \\
\text { marginales desde una perspectiva } \\
\text { geográfica y ambiental } \\
\text { (ii) Sentar las bases para analizar } \\
\text { las poblaciones marginales desde } \\
\text { una perspectiva genética. }\end{array}$ & Europa & $\begin{array}{l}\text { Pinus pinaster Ait. } \\
\text { Pinus halepensis Mill. } \\
\text { Pinus nigra Arnold. } \\
\text { Pinus pinea } \text { L. } \\
\text { Pinus sylvestris } \mathrm{L} . \\
\text { Abies alba } \text { Mill. } \\
\text { Picea abies } \mathrm{L} .\end{array}$ & $\begin{array}{l}\text { Serra-Varela } \\
\text { et al., en prep }\end{array}$ \\
\hline
\end{tabular}

En el primer trabajo, testamos el efecto de incorporar información molecular en los MDE y analizamos la relación entre la distancia genética, ambiental y geográfica. Para este objetivo, empleamos como especie modelo el pino marítimo (Pinus pinaster Ait.) compuesto por ocho grupos infra-específicos definidos a partir de criterios genéticos (ver Fig. la). En este trabajo, comparamos dos modelos de nicho incorporando y no incorporando información genética (ver Fig. 1b).

En el segundo trabajo, evaluamos la vulnerabilidad al cambio climático de dos emblemáticas coníferas Mediterráneas, el pino marítimo y el pino carrasco (Pinus halepensis Mill.) teniendo en cuenta su capacidad adaptativa, su exposición al cambio climático y la incertidumbre derivada de las proyecciones climáticas. Para ello, 
a)

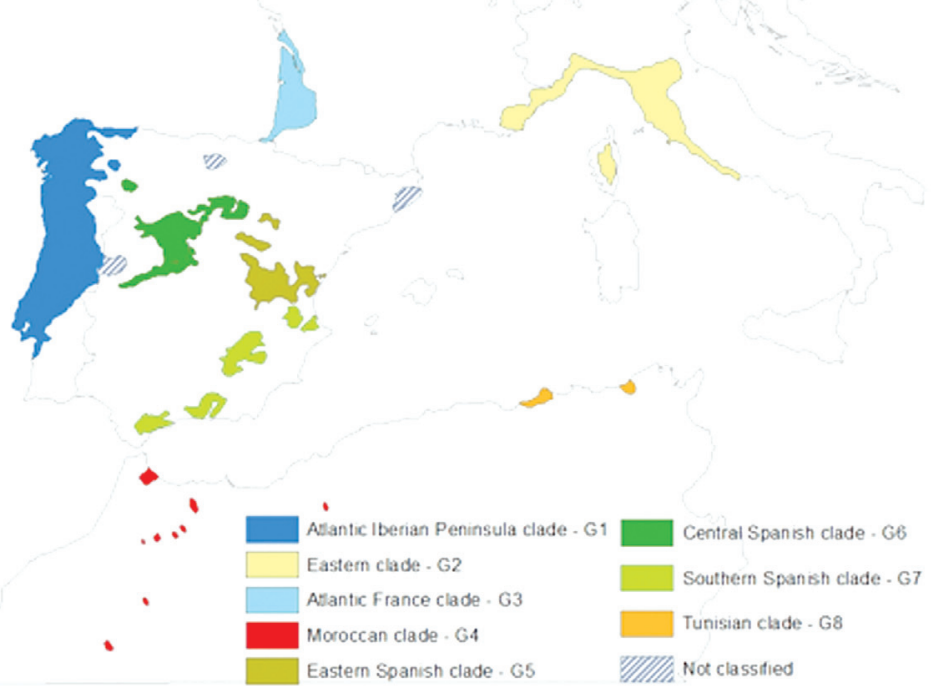

b)

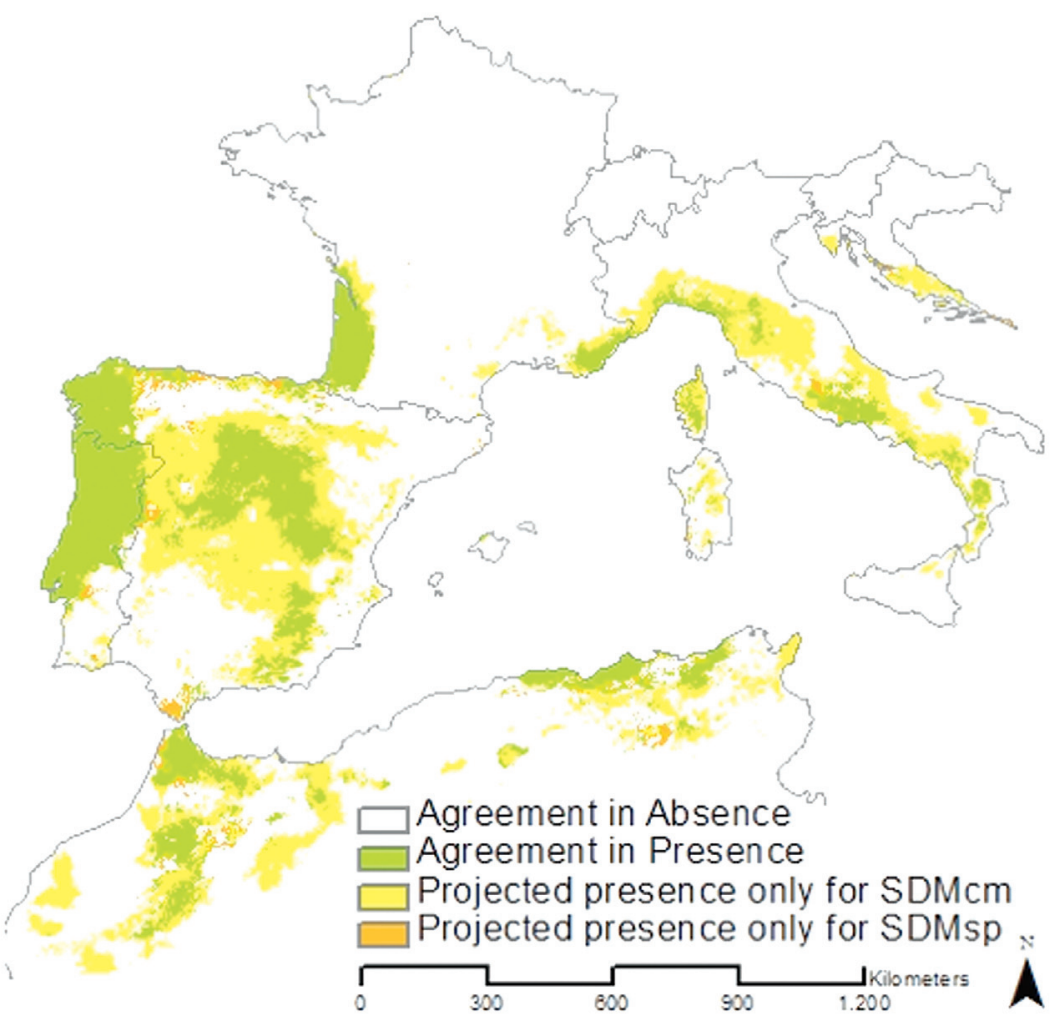

Figura 1. a) Grupos genéticos detectados para la especie Pinus pinaster Ait. b) Comparación del modelo que incorpora información genética (SDMcm) y el módelo clásico sin información genética (SDMsp). 
calibramos MDE incorporando información genética, los proyectamos a 42 predicciones climáticas diferentes correspondientes a 2050, de cara a incorporar la incertidumbre derivada de los escenarios climáticos futuros y evaluamos la exposición de las especies dependiendo de la concordancia entre las 42 proyecciones.

En tercer lugar, incorporamos en la evaluación de vulnerabilidad al cambio climático la exposición a factores bióticos. Para ello, usamos como especie modelo Pinus pinaster y una de sus potenciales enfermedades, el chancro resinoso, causado por el hongo Fusarium circinatum Nirenberg \& O'Donnell. Este estudio se desarrolló a nivel nacional (España) de cara a obtener resultados con alta calidad y resolución a partir de los datos disponibles para este territorio. Calibramos MDE para el pino y la enfermedad, proyectamos a 18 escenarios futuros representativos de 2050 y evaluamos la exposición del pino a factores abióticos (climáticos) y bióticos.

Finalmente, empleamos los MDEs para definir la marginalidad de una manera estandarizada y considerando los principales procesos que afectan al flujo genético. Para ello, se analizaron de manera independiente factores geográficos y ambientales, de cara a considerar los procesos de aislamiento por distancia y aislamiento por ambiente. Como especies modelos para el desarrollo de este trabajo, se emplearon siete coníferas europeas (cuatro de ellas Mediterráneas): Pinus halepensis Mill., Pinus pinaster Ait., Pinus nigra Arnold, Pinus pinea L., Pinus sylvestris L., Picea abies L. y Abies alba Mill., todas ellas caracterizadas genéticamente.

\section{Resultados y discusión}

Como resultado del primer trabajo, comprobamos una mejora significativa en las predicciones de los modelos que incorporaban información genética. La comparación de los modelos obtenidos se puede apreciar en la Fig. 1b, observándose mucho más realista la correspondiente al modelo que incorpora información genética $(\mathrm{SDMcm})$ dada la frugalidad del pino y las restricciones de carácter no climático que limitan su distribución.

A través del segundo trabajo pudimos plantear directrices de conservación específicas (in situ, ex situ o in situ con monitorización) para cada grupo infra-específico de las dos especies analizadas en base a una serie de criterios sencillos y objetivos (ver Tabla 2).

El tercer trabajo, realizado en una extensión menor y con mayor calidad y precisión en las fuentes de datos, permite detectar poblaciones amenazadas distinguiendo el factor principal de riesgo y planteando la mejor estrategia para cada caso dependiendo también de la aptitud productora o no de las masas (ver Fig. 3).

Para evaluar la marginalidad geográfica, se definieron cuatro índices caracterizando la posición de las poblaciones dentro de la mancha de distribución en la que encuentran, así como su posición con respecto al resto de la distribución de la especie (ver Tabla 3). Para evaluar la marginalidad ambiental, se empleó la probabi- 
Tabla 2. Recomendaciones de estrategias de conservación basadas en la distribución actual del grupo genético, la proyección actual de su modelo de distribución de especies - MDE y su aptitud climática futura.

\begin{tabular}{|c|c|c|l|}
\hline $\begin{array}{c}\text { Distribución } \\
\text { actual }\end{array}$ & $\begin{array}{c}\text { MDE - } \\
\text { Proyección actual }\end{array}$ & $\begin{array}{c}\text { Aptitud climática } \\
\text { futura - 2050 }\end{array}$ & \multicolumn{1}{|c|}{$\begin{array}{c}\text { Estrategia } \\
\text { de Conservación }\end{array}$} \\
\hline Presente & - & Probablemente no apto & ex situ. \\
\hline Presente & - & Probablemente apto & in situ con monitorización. \\
\hline Presente & - & Incierto & $\begin{array}{l}\text { in situ con un esquema de } \\
\text { monitorización intense y dirigida. }\end{array}$ \\
\hline Ausente & Apto & Probablemente apto & $\begin{array}{l}\text { Primera opción para translocación } \\
\text { en el momento actual. }\end{array}$ \\
\hline Ausente & Apto & Incierto & $\begin{array}{l}\text { Segunda opción para translocación } \\
\text { en el momento actual. }\end{array}$ \\
\hline Ausente & No apto & Probablemente apto & $\begin{array}{l}\text { Primera opción para translocación } \\
\text { en el medio plazo. }\end{array}$ \\
\hline Ausente & No apto & Incierto & $\begin{array}{l}\text { Segunda opción para traslocación } \\
\text { en el medio plazo. }\end{array}$ \\
\hline
\end{tabular}

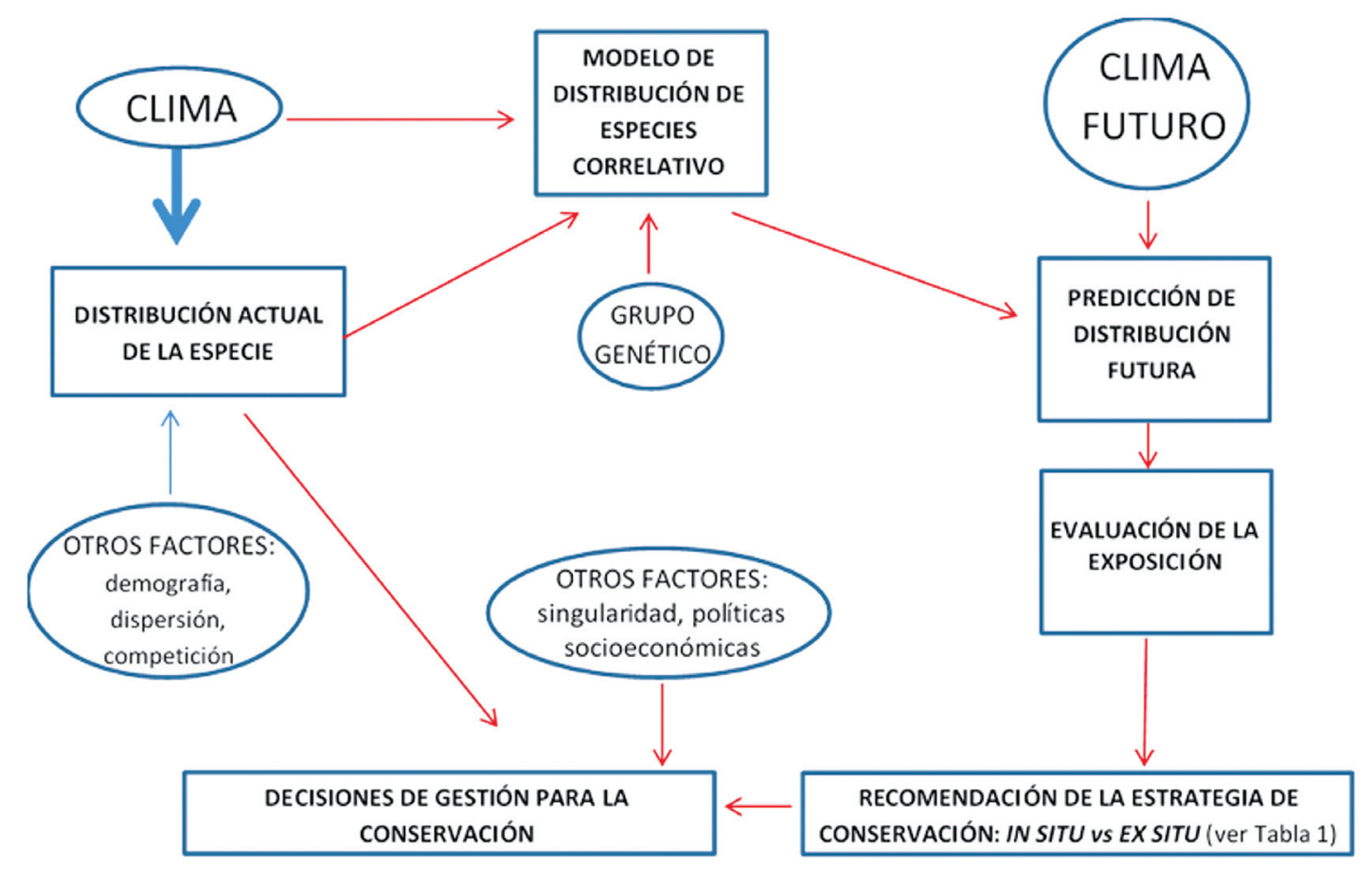

Figura 2. Marco para la toma de decisiones de conservación

lidad de adecuación ambiental estimada a través de MDEs. En todos los casos se analizaron poblaciones genéticamente caracterizadas de cara a continuar el estudio con una segunda fase en la que evaluaremos las consecuencias de la marginalidad en los patrones de diversidad genética. 

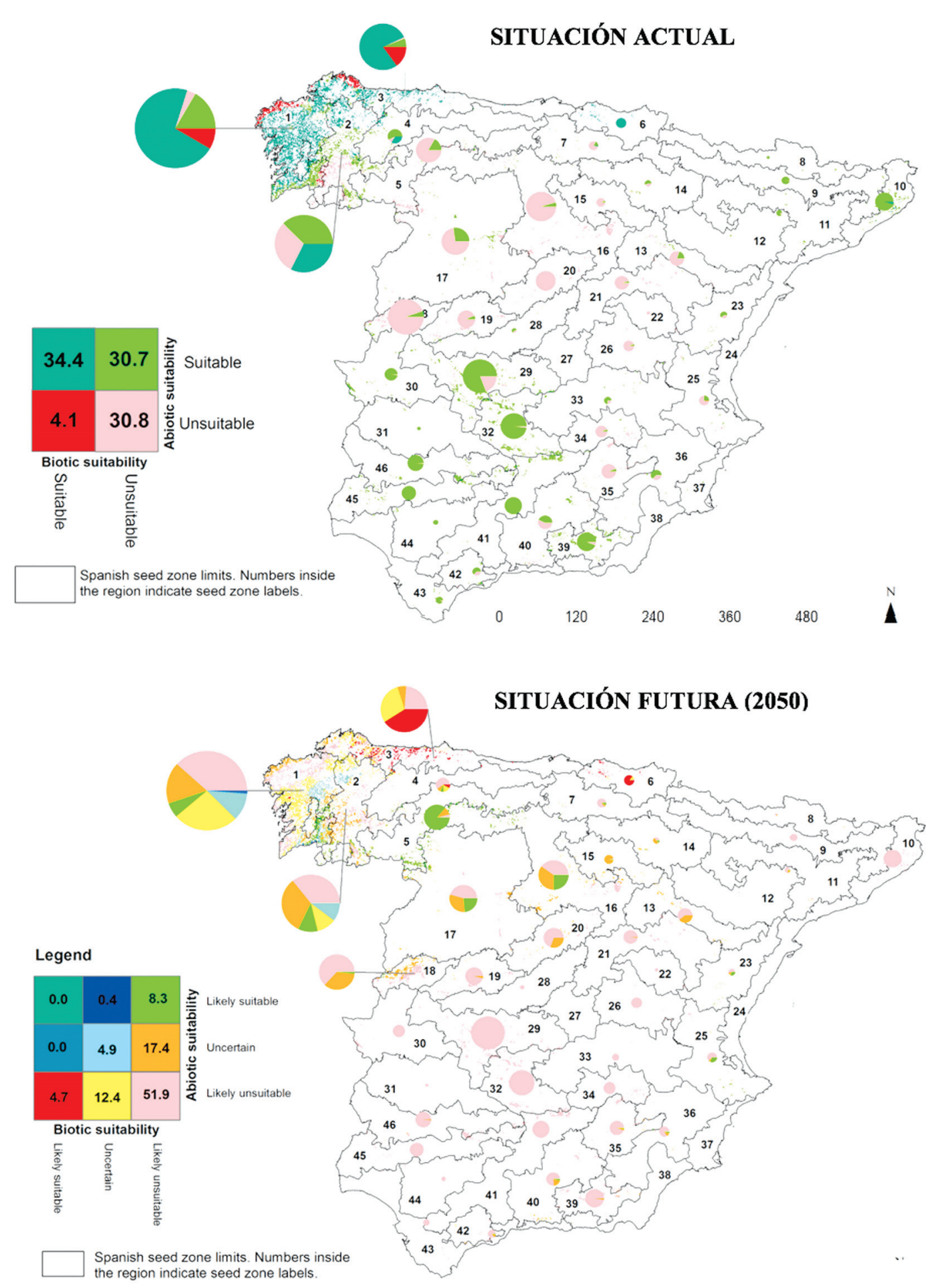

Figura 3. Evaluación actual y futura (2050) de la exposición abiótica y biótica de Pinus pinaster Ait. estimada a partir de los mapas de aptitud climática del pino en ambos momentos y de uno de sus patógenos, el chancro del pino. El tamaño de los gráficos es proporcional a la ocupación del pino en las regiones de identificación y utilización de semillas. Los números en la leyenda se corresponden con el porcentaje de la distribución del pino que presenta esa combinación de exposiciones. 
Tabla 3. Índices desarrollados para evaluar la marginalidad desde un punto de vista geográfico y ambiental. Los polígonos azules representan la distribución de la especie. Los puntos rojos son poblaciones genéticamente caracterizadas.

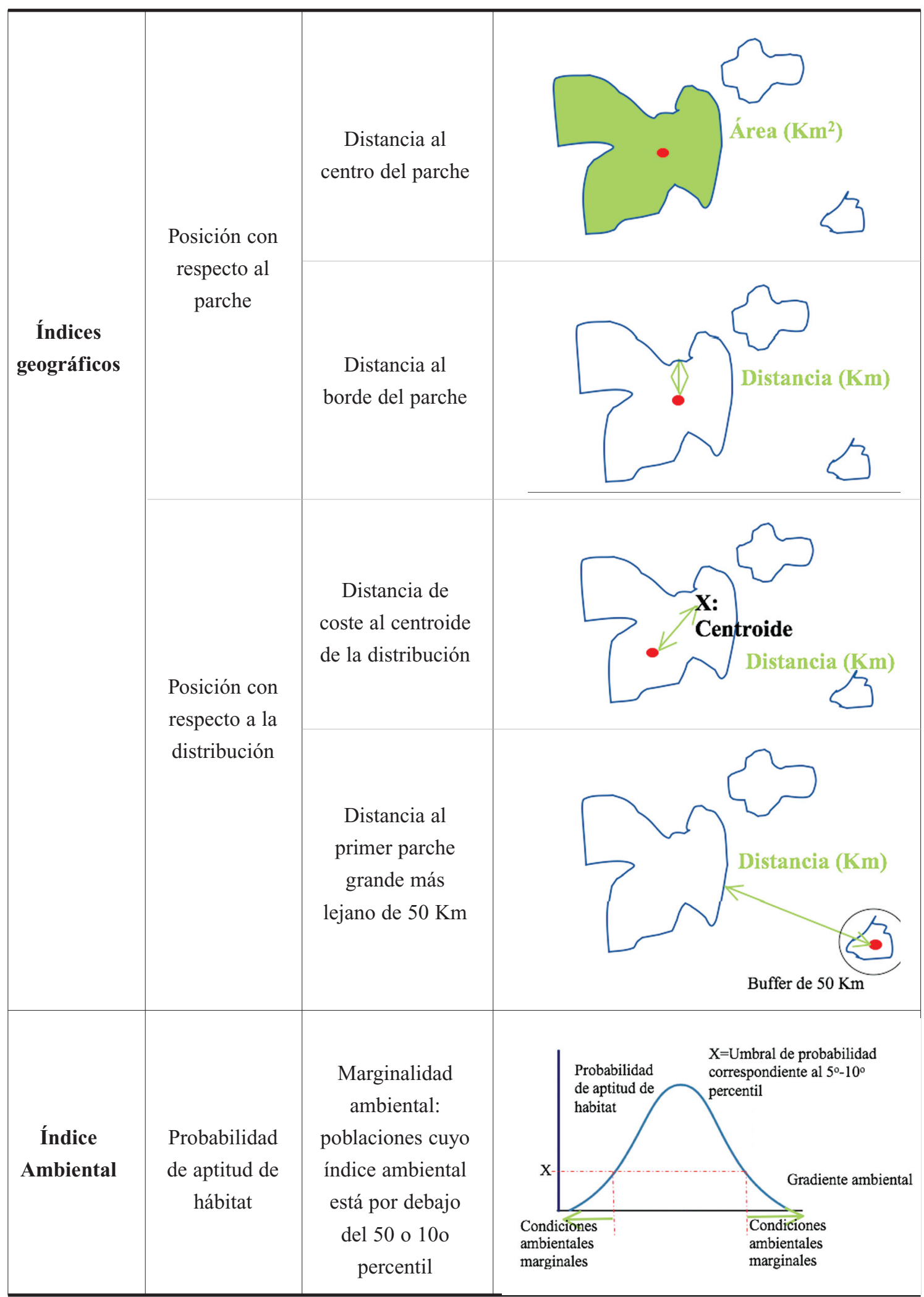


A lo largo de este trabajo, se solucionan con éxito las principales limitaciones de los MDE para su uso en la gestión para la conservación, permitiendo desarrollar aproximaciones de gran aplicación para el mundo forestal.

\section{Referencias bibliográficas}

Araújo, M.B. \& Luoto, M. (2007) The importance of biotic interactions for modelling species distributions under climate change. Global Ecology and Biogeography, 16, 743753. https://doi.org/10.1111/j.1466-8238.2007.00359.x

Fordham, D.A., Wigley, T.M.L. \& Brook, B.W. (2011) Multi-model climate projections for biodiversity risk assessments. Ecological Applications, 21, 3317-3331. https://doi.org/ 10.1890/11-0314.1

Guisan, A., Tingley, R., Baumgartner, J.B., Naujokaitis-Lewis, I., Sutcliffe, P.R., Tulloch, A.I.T., Regan, T.J., Brotons, L., McDonald-Madden, E., Mantyka-Pringle, C., Martin, T.G., Rhodes, J.R., Maggini, R., Setterfield, S. a, Elith, J., Schwartz, M.W., Wintle, B. A., Broennimann, O., Austin, M., Ferrier, S., Kearney, M.R., Possingham, H.P. \& Buckley, Y.M. (2013) Predicting species distributions for conservation decisions. Ecology letters, 16, 1424-1435. https://doi.org/10.1111/ele.12189

Guisan, A. \& Zimmermann, N.E. (2000) Predictive habitat distruction models in ecology. Ecological Modelling, 135, 147-186. https://doi.org/10.1016/S0304-3800(00)00354-9

IPCC (2007) Climate Change 2007: impacts, adaptation and vulnerability: contribution of Working Group II to the fourth assessment report of the Intergovernmental Panel, Cambridge.

Lefèvre, F., Koskela, J., Hubert, J., Kraigher, H., Longauer, R., Olrik, D.C., Schueler, S., Bozzano, M., Alizoti, P., Bakys, R., Baldwin, C., Ballian, D., Black-Samuelsson, S., Bednarova, D., Bordács, S., Collin, E., de Cuyper, B., de Vries, S.M.G., Eysteinsson, T., Frýdl, J., Haverkamp, M., Ivankovic, M., Konrad, H., Koziol, C., Maaten, T., Notivol Paino, E., Oztürk, H., Pandeva, I.D., Parnuta, G., Pilipovic, A., Postolache, D., Ryan, C., Steffenrem, A., Varela, M.C., Vessella, F., Volosyanchuk, R.T., Westergren, M., Wolter, F., Yrjänä, L. \& Zarija, I. (2013) Dynamic conservation of forest genetic resources in 33 European countries. Conservation biology: the journal of the Society for Conservation Biology, 27, 373-84. https://doi.org/10.1111/j.1523-1739.2012.01961.x

Matyas, C., Nagy, L. \& Jarmay, E.U. (2009) Genetic background of response of trees to aridification at the xeric forest limit and consequences for bioclimatic modelling. Bioclimatology and Natural Hazards, pp. 179-196. Springer, Netherlands.

Myers, N., Mittermeier, R.A., Mittermeier, C.G., da Fonseca, A.B. \& Kent, J. (2000) Biodiversity hotspots for conservation priorities. Nature, 403, 72. https://doi.org/10.1038/35 002501

Nicotra, A.B., Beever, E.A., Robertson, A.L., Hofmann, G.E. \& O’Leary, J. (2015) Assessing the components of adaptive capacity to improve conservation and management efforts under global change. Conservation Biology, 29, 1268-1278. https://doi.org/ 10.1111/cobi.12522

Schoville, S.D., Bonin, A., François, O., Lobreaux, S., Melodelima, C. \& Manel, S. (2012) Adaptive Genetic Variation on the Landscape: Methods and Cases. Annual Review of 
Ecology, Evolution, and Systematics, 43, 23-43. https://doi.org/10.1146/annurevecolsys-110411-160248

Serra-Varela, M.J., Alía, R., Pórtoles, J., Gonzalo-Jiménez, J., Soliño, M., Grivet, D. \& Raposo, R. (2017a) Incorporating exposure to pitch canker disease to support management decisions of Pinus pinaster Ait. in the face of climate change. PloS one, 12. https://doi.org/10.1371/journal.pone.0171549

Serra-Varela, M.J., Alía, R., Ruíz-Daniels, R., Zimmermann, N.E., Gonzalo-Jiménez, J. \& Grivet, D. (2017b) Assessing vulnerability of two Mediterranean conifers to support genetic conservation management. Diversity and Distributions. https://doi.org/10. 1111/ddi.12544

Serra-Varela, M.J., Grivet, D., Vincenot, L., Broennimann, O., Gonzalo-Jiménez, J. \& Zimmermann, N.E. (2015) Does phylogeographic structure relate to climatic niche divergence? A test using maritime pine (Pinus pinaster Ait.). Global Ecology and Biogeography, 24, 1302-1313. https://doi.org/10.1111/geb.12369

Thomassen, H.A., Cheviron, Z.A., Freedman, A.H., Harrigan, R.J., Wayne, R.K. \& Smith, T.B. (2010) Spatial modelling and landscape-level approaches for visualizing intraspecific variation. Molecular Ecology, 19, 3532-3548. https://doi.org/10.1111/j.1365294X.2010.04737.x

Thuiller, W., Albert, C., Araújo, M.B., Berry, P.M., Cabeza, M., Guisan, A., Hickler, T., Midgley, G.F., Paterson, J., Schurr, F.M., Sykes, M.T. \& Zimmermann, N.E. (2008) Predicting global change impacts on plant species' distributions: Future challenges. Perspectives in Plant Ecology, Evolution and Systematics, 9, 137-152. https://doi.org/10.1016/ j.ppees.2007.09.004 


\section{Datos de la tesis}

Premio Universitario de la SECF 2017 a la mejor tesis doctoral.

Directores: Delphine Grivet y Julián Gonzalo.

Tutor: Ricardo Alía.

Centro: Instituto de Investigación en Gestión Forestal Sostenible. INIA

Fecha de defensa: Enero de 2017

\section{Publicaciones derivadas de la tesis doctoral}

Serra-Varela, M.J., Alía, R., Pórtoles, J., Gonzalo-Jiménez, J., Soliño, M., Grivet, D. \& Raposo, R. (2017a) Incorporating exposure to pitch canker disease to support management decisions of Pinus pinaster Ait. in the face of climate change. PloS one, 12.

Serra-Varela, M.J., Alía, R., Ruíz-Daniels, R., Zimmermann, N.E., Gonzalo-Jiménez, J. \& Grivet, D. (2017b) Assessing vulnerability of two Mediterranean conifers to support genetic conservation management. Diversity and Distributions.

Serra-Varela, M.J., Grivet, D., Vincenot, L., Broennimann, O., Gonzalo-Jiménez, J. \& Zimmermann, N.E. (2015) Does phylogeographic structure relate to climatic niche divergence? A test using maritime pine (Pinus pinaster Ait.). Global Ecology and Biogeography, 24, 1302-1313. 